\title{
Erratum to: Molecular study of Trypanosoma caninum isolates based on different genetic markers
}

\author{
Juliana H. S. Barros • Helena K. Toma • Maria de Fatima Madeira
}

Published online: 27 January 2015

(C) Springer-Verlag Berlin Heidelberg 2015

\section{Erratum to: Parasitology Research}

DOI 10.1007/s00436-014-4291-0

The Table 1 of the original publication of this article contained a mistake in the alignment of the primers. Below is the correct presentation.
Table 1 Molecular targets used for amplification of different genomic regions of Trypanosoma caninum

\begin{tabular}{lll}
\hline Target & Primers & Sequence 5'-3' \\
\hline 18S rDNA & TRY927F & GAAACAAGAAACACGGGAG \\
& TRY927R & CTACTGGGCAGCTTGGA \\
& SSU561F & TGGGATAACAAAGGAGCA \\
& SSU561R & CTGAGACTGTAACCTCAAAGC \\
24S $\alpha$ rDNA & D75 & GCAGATCTTGGTTGGCGTAG \\
& D76 & GGTTCTCTGTTGCCCCTTTT \\
Cytochrome & P18 & GACAGGATTGAGAAGCGAGAGAG \\
b & P20 & CAAACCTATCACAAAAAGCATCTG \\
kDNA & 121 & AAATAATGTACGGGKGAGATGCATGA \\
& 122 & GGTTCGATTGGGGTTGGTGTATATA \\
ITS1 rDNA & KIN1 & GCGTTCAAAGATTGGGCAAT \\
& KIN2 & CGCCCGAAAGTTCACC \\
\hline
\end{tabular}

The online version of the original article can be found at http://dx.doi.org/ 10.1007/s00436-014-4291-0.

J. H. S. Barros

Programa de Pós-Graduação em Pesquisa Clínica em Doenças Infecciosas, Instituto de Pesquisa Clínica Evandro Chagas, Fundação Oswaldo Cruz, Av. Brasil 4365, Rio de Janeiro, RJ 21040-900, Brazil

H. K. Toma

Laboratório de Diagnóstico Molecular e Hematologia, Faculdade de Farmácia, Universidade Federal do Rio de Janeiro, Rio de

Janeiro, Brazil

M. de Fatima Madeira $(\bowtie)$

Laboratório de Vigilância em Leishmanioses, Instituto de Pesquisa

Clínica Evandro Chagas, Fundação Oswaldo Cruz, Av. Brasil 4365,

Rio de Janeiro, RJ 21040-900, Brazil

e-mail: fatima.madeira@ipec.fiocruz.br 